\title{
Research on Green Transport Mode of Chinese Bulk Cargo Based on Fourth-Party Logistics
}

\author{
Jixiao Wu $(\mathbb{D}$, Yinghui Wang $(\mathbb{D}$, Wenlu Li $(\mathbb{D}$, and Haixia Wu $(\mathbb{D}$ \\ Department of Economics and Management, Shijiazhuang Tiedao University, Shijiazhuang/050043, China \\ Correspondence should be addressed to Yinghui Wang; wang112111@163.com
}

Received 4 September 2021; Revised 15 October 2021; Accepted 5 November 2021; Published 24 November 2021

Academic Editor: Eduardo Lalla-Ruiz

Copyright ( $) 2021$ Jixiao Wu et al. This is an open access article distributed under the Creative Commons Attribution License, which permits unrestricted use, distribution, and reproduction in any medium, provided the original work is properly cited.

\begin{abstract}
Due to the problems such as the excessive proportion of road transport and extreme carbon emission situation of China's transport structure adjustment, this paper combines the fourth-party logistics with the bulk cargo green transport. It is advancing the adjustment of China's bulk cargo transport structure using fourth-party logistics. This paper improves the particle swarm optimization algorithm to compare the integrated cost and carbon emissions of different bulk fourth-party transport networks to verify the benefits of the fourth-party logistics on bulk cargo transport networks' cost reduction and emission reduction. The results show that using the fourth-party logistics model to promote the transfer of cargoes from road to rail can reduce the integrated cost of the transport network, reduce carbon emissions, and achieve green transport.
\end{abstract}

\section{Introduction}

Since the signing of the Paris Agreement, China has been actively engaged in global emission reductions and has taken the initiative to assume responsibility for emission reductions [1]. According to the China Statistical Yearbook data, the transportation industry is one of China's fastest-growing carbon emissions industries. Between 2000 and 2015, the total carbon emissions of China's transportation industry increased from 58.22 million tons to 190.1 million tons. It increased by a full 2.26 times, with an average annual growth rate of $7.9 \%$ [2]. Reducing the carbon emissions in the transportation industry is of considerable significance to China's modern integrated transportation system and green transport with "high energy efficiency, low pollution, low consumption, and low emissions" [2]. Transport freight volume is closely related to carbon emissions: in 2019, China's road freight volume reached 41.6064 billion tons, rail freight volume has reached 4.38904 billion tons, and road freight volume has reached 9.48 times the railway freight volume [2]. According to the US. Federal Railway Administration report, the carbon emissions from road transport at the same distance are nearly four times that of rail transport [3]. The above data show that the high proportion of road freight is an essential reason for the high carbon emissions of China's transportation industry. If it can give full play to the advantages of railways in reducing carbon emissions and implement the Three-Year Action Plan issued by the State Council of China to guide the transfer of cargo from road transport to railway transport as soon as possible, it will greatly benefit the realization of China's sustainable development strategy and the development of green transport.

To promote the adjustment of transport structure, reduce the carbon emissions of transport, improve the integrated transport efficiency, and reduce the cost of logistics, the General Office of the State Council issued the Three-Year Action Plan for Promoting the Adjustment of the Transport Structure (2018-2020) in October 2018, which proposed the action of upgrading the capacity of railway transport, and made it clear that promoting the "road to rail" of bulk cargo transport is the main direction of transport supply-side reform [4]. In September 2019, China's National Development and Reform Commission also cooperated with relevant departments and issued the "Guiding Opinions on Accelerating the Construction of Railway Special Lines," which aim to solve the "first and last kilometre" problem of the bulk cargo of railway transport and to provide solutions 
to the distribution problem of "road to rail" [5]. In addition, the State Railway Administration of China has taken a number of measures in railway logistics infrastructure, railway transport prices, and preferential transport policies to deepen the reform of railway freight transport and accelerate the process of green transport.

Recently, the traditional bulk cargo transportation industry has gotten rid of the low-price-first model. Shippers' demand for the safety, convenience, and delivery time of transport is increasingly higher. The transportation industry urgently needs a new model to improve the economic benefit. In order to optimize transport costs under lowcarbon emissions, the following innovations are made in this paper. (1) The fourth-party logistics promotes the rapid transfer of bulk cargo from road transport to railway transport, improves transport efficiency, constructs an intelligent transportation system, and optimizes the integrated cost of bulk cargo transport. (2) Unlike traditional bulk cargo transport, this paper focuses on the transport process between multiple shippers and freight enterprises. Moreover, the improved particle swarm optimization algorithm compares the integrated cost and carbon emissions of road and rail transport under different conditions within a limited time.

The remainder of the paper is structured in the following manner. Section 2 presents an overview of the relevant literature, focusing on green transport, bulk cargo transport, fourth-party logistics, and algorithm design. Section 3 builds the optimization model of bulk cargo green transport mode based on fourth-party logistics, while Section 4 uses different experiments to illustrate the optimization effect of the fourth-party logistics of bulk cargoes on green transport. Section 5 summarizes the findings and outlines potential future research extensions.

\section{Literature Review}

In order to promote the development of green transport, researchers have carried out much research on the lowcarbon emission reduction of the transportation industry. They analyzed the local transportation system through the CUTE matrix and pointed out that the implementation of low-carbon policies and improvement of transportation technology are essential means to reduce the carbon emissions of the transportation industry [6]. A model of urban vehicle distribution with low carbon, energy saving, and low cost was constructed, and genetic algorithms were designed to optimize vehicle distribution routes [7]. Measured the carbon emissions of the integrated logistics system of the port and proved that the carbon emissions of transport, heavy equipment and logistics services account for a significant proportion of the transportation system [8]. They focused on the impact of carbon taxes on different transport modes and compared the carbon emissions and economic benefits of China's railways, roads, and waterways through a general equilibrium model [9]. The above researchers' research on low-carbon emissions provides an important idea for this article to explore green logistics. However, there are still not many studies on promoting green logistics in academia, and it needs to be further improved.

In analyzing the characteristics of bulk cargo and promoting the green transport of bulk cargo, researchers based on the case of coal railway transport networks used bi-level optimization to explore the route of railway freight under the condition of network interference [10]. They used a complex network to evaluate the freight capacity of China's road and railway nodes and explored the differences in the transportation of bulk cargoes by road and railway [11]. Combine the bulk cargo price prediction model with neural network algorithms to improve railway freight volume prediction speed and accuracy [12]. They also combined the TOPSIS method to analyze the efficiency of bulk cargo transport in the Brazilian railway system, proposed some methods for optimizing the rail transport of bulk cargoes, and improved rail freight efficiency [13]. However, researchers' research on bulk cargoes mostly focuses on water transport, and the research on rail transport of bulk cargoes needs further innovation.

In the research of transport informatization: researchers combined the views of 48 experts from 20 countries to explore the supply chain management model of the future transportation industry and logistics industry from the five Delphi methods of energy emissions, consumer behavior, future transportation mode, future supply design, and innovation and concluded that a supply chain management model that can help decision making, information sharing, and sustainability would emerge in the future and the fourth-party logistics could provide this management model [14]. They proposed a discrete flow model to describe the reverse logistics supply chain of remanufactured products, which provided some ideas for the research of fourth-party logistics [15]. Constructed an " $N+1+N$ " fourth-party logistics model based on China's "VAT Reform" policy to achieve overall symmetry in logistics, business, information, and capital flows and explored the fourth-party logistics profit model [16]. Combined with Internet technology, it integrated financial institutions, consulting companies, and other industries into the logistics industry chain to promote the development of industry informatization and improve the efficiency of logistics industry operation [17]. Researchers integrated the government procurement information network through cloud technology to improve government procurement efficiency and optimise supply chain management [18]. The above researchers' research on the combination of logistics and big data provides many ideas for the research of fourth-party logistics in this paper. However, the existing logistics informatization research mostly focuses on emergency logistics, and the research on low-carbon logistics is not much.

In the process of studying the integrated cost of the transport network and the optimal vehicle configuration, researchers incorporated the cost of risk management and the psychological cost of waiting for rescue into the emergency reverse logistics model, constructed the logistics network model, and optimized the integrated cost of transport network based on the trade-off of three conflicting objectives: logistics cost, risk cost, and psychological cost 
[19]. They improved the multiobjective particle swarm optimization algorithm to solve the multiobjective enterprises under the carbon tax policy [20]. and improved the genetic algorithm to optimize the transportation network's cost and distribution of the vehicle [21]. By studying these algorithms, this paper finally chooses to use the improved particle swarm optimization algorithm to solve the transport network optimization problem between multiple shippers and consignees. Influenced by the relevant policies and environmental protection requirements, China's research on bulk cargo transport structural adjustment has achieved certain results. It has become an inevitable trend to shift the mode of transport of cargo from road to rail transport. However, it can be seen from the above literature that researchers still have doubts about how to promote the "road to rail" of bulk cargo and reduce the integrated cost of the transportation industry. For the optimization of the low-carbon transport between multiple shippers and multiple consignees, the existing research needs to be further deepened. To this end, this paper innovatively combines fourth-party logistics with bulk cargo green transport between multiple shippers and multiple consignees. Through the intelligent bulk cargo fourth-party logistics network to attract more sources and reduce transportation cost, improve the particle swarm optimization algorithm to evaluate the fourth-party logistics network's effect to prove the fourth-party logistics' optimization effect.

\section{Green Transport Mode of Bulk Cargoes Based on Fourth-Party Logistics}

According to the definition of the China Railway Customer Service Center, bulk cargo is large cargo volume and stable cargo, such as coal, coke, petroleum, and metal ore, which can be determined in advance for transportation needs. Bulk cargo transport accounts for more than $50 \%$ of China's cargo transport market, ranking first among all types of freight transport [3]. The proportion of bulk cargo transport shared by roads is up to $75.06 \%$ [22]. Due to the differences in transport means, energy, capacity, and facilities, rail transport's energy consumption and emission intensities are only $1 / 7$ and $1 / 13$ of road transport [22]. Carbon emissions are higher in road transport and lower in railway transport, so the implementation of "road to rail" to promote the transfer of bulk cargo from road transport to rail transport has brought significant energy conservation and emission reduction effects (unit turnover can save $86 \%$ of energy consumption and reduce $92 \%$ of energy emissions [23]). However, railway bulk cargo transport is still challenging to complete "point-to-point, door-to-door" transport. The transport process is complicated, and the transaction costs are high. Therefore, it is necessary to promote the transfer of bulk cargo transport from road transport to railway transport.

Based on the existing research related to logistics informatization [24-28], this paper builds a green transport mode of bulk cargo through fourth-party logistics. Compared with other researchers' big data models, this paper incorporates carbon emission cost factors into the selection and evaluation system of bulk cargo transport methods. It determines the optimal transport model by comparing the integrated cost and carbon emissions between different transport modes within a limited time.

3.1. Problem Description and Assumptions. Compared with road transport, railway transport has advantages of low cost, large volume, all-weather, energy-saving and environmental protection, safety, and stability. There are also many shortcomings such as complicated transport links, high costs for short-distance freight, tight local transport capacity, limited timeliness, and insufficient flexibility [29]. In this paper, the cost of fourth-party logistics and carbon emissions is included in the green transport mode of bulk cargoes and the optimal transport method is selected by comparing different transport costs and transport efficiencies.

In the traditional bulk cargo transport network, due to insufficient informationization, inflexibility, inadequate policy support, and imperfect multimodal transport mechanism, bulk cargo rail transport is complicated, and the transaction procedures are numerous [30]. The lack of communication between shipper and cargo enterprise, between consignee and cargo enterprise, and between shipper and consignee created a large amount of information game cost, which makes shipper more willing to choose road rather than railway transport to bear the volume of bulk cargo. As a result, in promoting the green transportation of bulk goods from road to railway, a big data logistics service that can share transport network information, simplify transaction procedures, and promote low-carbon emissions is needed. Fourth-party logistics can provide such logistics services.

The use of fourth-party logistics to promote green transport of bulk cargoes has the following effects. (1) It is conducive to the application of intelligence, informatization, and big data. (2) It simplifies the railway transport process and improves the service level of multimodal transport with the railway as the core. (3) It reduces the integrated cost of green transport networks for bulk cargoes.

Figure 1 shows the design of the fourth-party logistics network for bulk cargo. In the fourth-party logistics network, shippers release logistics orders through the fourthparty logistics. The platform formulates transport plans after collecting detailed logistics information and distributes the information to the corresponding third-party logistics enterprises through bidding. Enterprises integrate the goods of different shippers through fourth-party logistics and transport the integrated goods according to the requirements of time, destination, and route. In this transport process, the enterprise enjoys certain autonomy. It must follow the relevant transport requirements and time limit requirements while undertaking logistics orders and is responsible for the transport process. When the order reaches the designated consignee, the driver will give feedback to the platform, and the task will end after confirmation by the platform. During this period, all businesses will be 
completed through the fourth-party logistics platform. After receiving the relevant logistics task instructions, each member should prepare in accordance with the transport plan formulated by the platform. Each member can communicate in real time and supervise the order process through the fourth-party logistics platform during the transport process. If there is any loss or accident in the transport process, the fourth-party logistics platform shall coordinate by relevant rules.

Figure 2 shows the schematic diagram of the fourthparty logistics network for bulk cargo. The fourth-party logistics is responsible for the bulk cargo delivery of products between multiple supply and demand point pairs of products. The bulk cargo is transported to different consignees $i$ through $n$ freight enterprises, where each freight enterprise is responsible for providing transport services to multiple supply and demand point pairs. The nodes in the network include shippers, consignees, and freight enterprises. Cargoes are sent from shippers and transferred to individual consignees by freight enterprises. $x_{i j k n}$ indicates the transport route from shipper $j$ to consignee $i$. In order to maximize the profits of the overall logistics network, it is necessary to reasonably plan the bulk cargo transport routes and the node distribution locations to minimize the sum of the costs of all the distribution routes $x_{i j k n}$. It is crucial to comprehensively consider the carbon emission and establish the green transport network model of bulk cargoes based on fourth-party logistics to minimize the integrated cost within a limited time.

The carbon emission cost of the logistics network involved in this paper mainly refers to the carbon dioxide emission cost generated by road or rail transport during the mainline transport process of the bulk cargo logistics network. For the convenience of calculation, this paper separately sets the cost of carbon emission factor per unit distance for road and rail transport $\alpha_{1}, \alpha_{2}$ [20] and calculates the overall carbon tax cost of logistics network without detailed calculation of the carbon emissions of a specific model. In other words, this paper takes the cost of carbon emission, the starting cost of the service, distribution of the cost of processing, and transportation as the total cost and finds the integrated cost of the logistics network when the total is minimized. This design avoids the problem of excessive objective functions in most existing low-carbon transport optimization studies. It is more suitable for the fourth-party logistics network than the single-objective optimization model.

In order to simplify the calculation process, this paper makes the following assumptions. (1) The transport time of the road in the transport network of the same condition is the same as that of the railway. (2) The fourthparty logistics needs additional opening costs, reducing the transport network's carbon tax rate and information cost after opening.
3.2. Description of Model Components. The relevant parameters, sets, and decision variables involved in this model are shown in Table 1.

3.3. Model Building. Based on the above symbol descriptions and variable definitions, the integrated cost and carbon emission of the fourth-party logistics network is shown as follows:

$$
\begin{aligned}
\operatorname{Min} C= & \sum y_{n}\left(H+C_{I}\right)+\sum_{i \in V} \sum_{j \in V} \sum_{n \in N} \sum_{k \in K} C_{n} x_{i j k n} \\
& +\sum_{i \in V} \sum_{j \in V} \sum_{n \in N} \sum_{k \in K} c_{i j k n} x_{i j k n}\left(d_{j n}^{1}+d_{i n}^{2}\right)+\alpha_{1,2} E \\
E= & \sum_{i \in V} \sum_{j \in V} \sum_{n \in V} \sum_{k \in K} \beta_{1,2} q_{i j k n} b_{n}^{p}\left(d_{j n}^{1}+d_{i n}^{2}\right) .
\end{aligned}
$$

The objective function formula (1) represents the optimal integrated cost of the transport network in a limited time, which includes the opening cost of the services of freight enterprises, loading and unloading costs of bulk cargo transport, transport costs, and the carbon emission cost incurred by vehicles of freight enterprises. Formula (2) represents carbon emissions from the transport network.

$$
\begin{aligned}
& \sum_{j \in N \cup} \sum_{n \in N} \sum_{k \in K} x_{i j k n}=b_{n}^{p}, \quad \forall i \in I, \forall p \in P, \\
& \sum_{j \in N \cup} \sum_{n \in N} \sum_{k \in K} x_{i j k n}=b_{n}^{p}, \quad \forall i \in I, \forall p \in P, \\
& \sum_{j \in N} \sum_{n \in N} \sum_{k \in K} x_{i j k n}=1, \quad \forall i \in I, \forall p \in P, \\
& \sum_{i \in N} \sum_{n \in N} \sum_{k \in K} x_{i j k n}=1, \quad \forall j \in J, \forall p \in P, \\
& \sum_{j \in N} \sum_{n \in N} \sum_{k \in K} a_{i j k n}^{p}=1, \quad \forall i \in I, \forall p \in P, \\
& \sum_{i \in N} \sum_{n \in N} \sum_{k \in K} a_{i j k n}^{p}=1, \quad \forall i \in I, \forall p \in P .
\end{aligned}
$$

Constraints (3) and (4) indicate that if the freight enterprise $n$ of the logistics network is selected to serve the supply point pair $p$, there must be a transport mode connected to it to transport the cargo; otherwise, no freight enterprise or transport mode is selected. Constraints (5) and (6) require that the bulk cargo be transported from shipper $j$ to consignee $i$ via freight enterprise $n$. Constraints (7) and (8) require, respectively, that the shipper $j$ and consignee $i$ of point of supply and demand point pair $p$ must transport the products via freight enterprise $n$. 


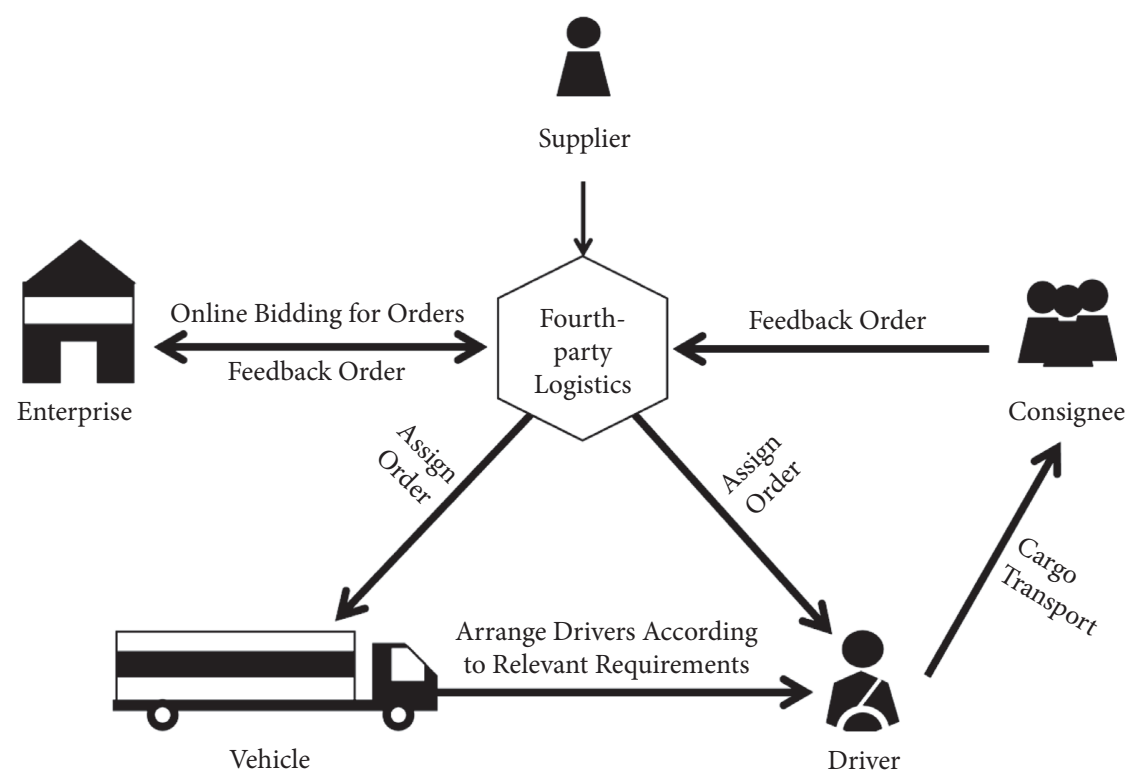

FIGURE 1: Bulk cargo fourth-party logistics network design.

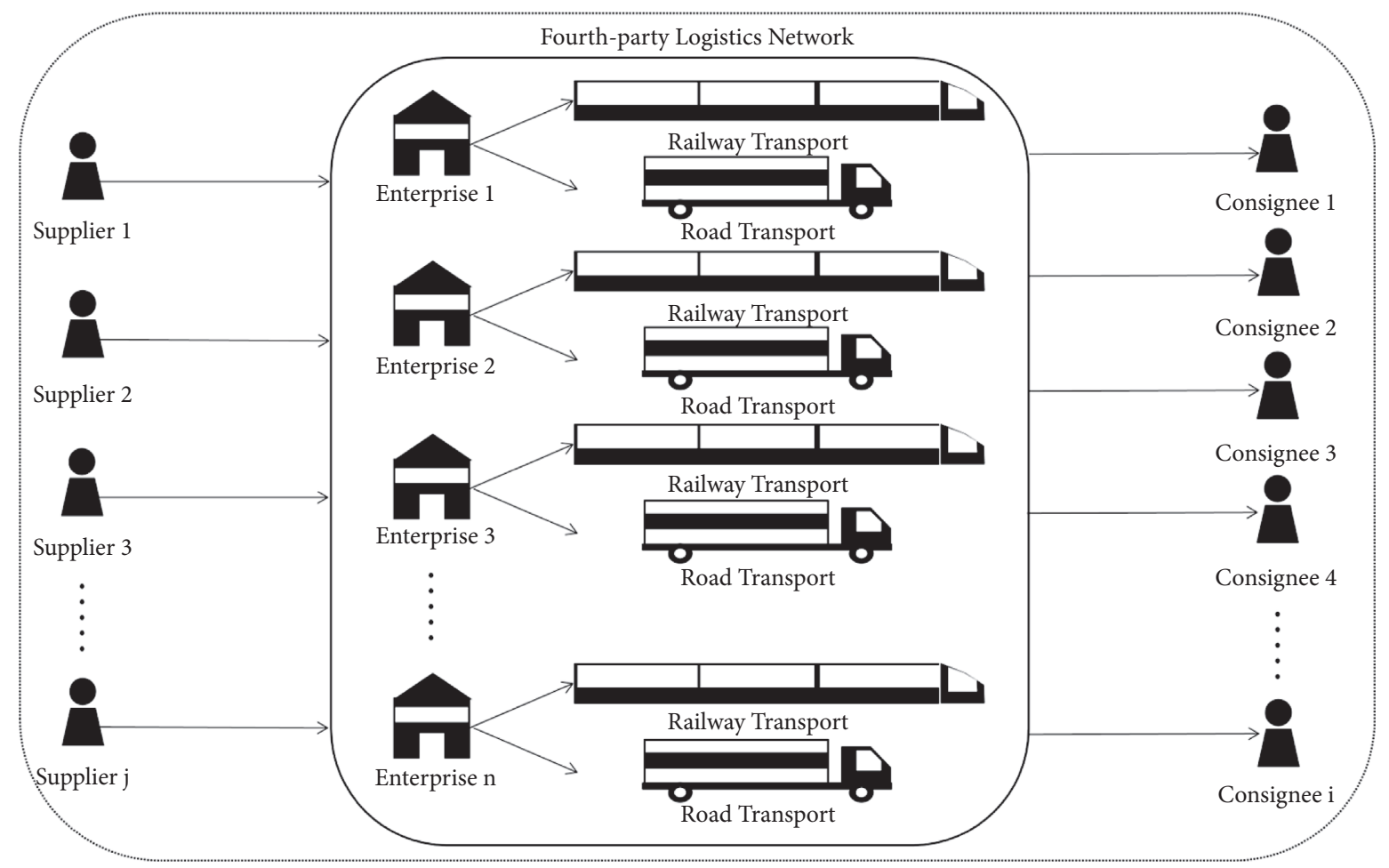

FIgURE 2: The fourth-party logistics network for bulk cargo.

$\beta_{1,2} \sum_{p \in P} b_{n}^{p} d^{p} \leq Q_{n} y_{n}, \quad \forall n \in N$

$\sum_{p \in P} a_{i j k n}^{p} d^{p} \leq q_{i j k n} x_{i j k n}, \quad \forall i \in V, \forall j \in V, \forall k \in K, \forall n \in N$,

$$
\beta_{1,2}\left(\sum_{i \in V} \sum_{j \in V} \sum_{n \in N} \sum_{k \in K} x_{i j k n}\right)=\sum_{i \in I} \sum_{j \in J} \sum_{n \in N} \sum_{k \in K} a_{i j k n}^{p},
$$

$$
\sum_{i \in I} S_{i}=\sum_{j \in J} D_{j}
$$


TABLE 1: Parameters, sets, and decision variables.

\begin{tabular}{|c|c|}
\hline Symbol & Description \\
\hline$d_{\text {in }}^{1}$ & The distance from the shipper $j$ to the freight $n(\mathrm{~km})$ \\
\hline$d_{i n}^{2 n}$ & The distance from the consignee $i$ to the freight $n(\mathrm{~km})$ \\
\hline$q_{i j k n}$ & $\begin{array}{c}\text { The unit transportation capacity of the vehicle } k \text { from the shipper } j \text { to the consignee } i \text { through the } \\
\text { freight enterprise } n\end{array}$ \\
\hline$c_{i j k n}$ & The cost of per vehicle per unit distance (yuan $/ \mathrm{km})$ \\
\hline$I^{\ln n}$ & The set including suppliers indexed by $i, I=\{0,1,2, \ldots, i\}$ \\
\hline$J$ & The set including shippers indexed by $j, J=\{0,1,2, \ldots, j\}$ \\
\hline$P$ & The set including supply and demand points from shipper $j$ to consignee $i$ \\
\hline$K$ & The set including freight vehicles indexed by $k$ \\
\hline$N$ & The set including freight enterprises indexed by $n, N=\{0,1,2, \ldots, n\}$ \\
\hline$G_{n}$ & Maximum load of the freight enterprise $n$ \\
\hline$C_{n}$ & The cost of loading and unloading cargo of the freight enterprise $n$ per unit time \\
\hline$S_{i}$ & The set including cargo quantity obtained by the consignee $i$ \\
\hline$D_{j}$ & The set including cargo quantity issued by the shipper $j$ \\
\hline$W_{n}$ & The freight handling capacity of the freight enterprise $n$ \\
\hline$\alpha_{1}, \alpha_{2}$ & The carbon tax rate of bulk cargo transport network with/without fourth-party logistics \\
\hline$\beta_{1}, \beta_{2}$ & The carbon emission factors per unit distance per product in road/rail transport \\
\hline$d^{p}$ & The number of emergency supplies between the point pair $p$ in the period, $p \in P$ \\
\hline \multicolumn{2}{|r|}{ 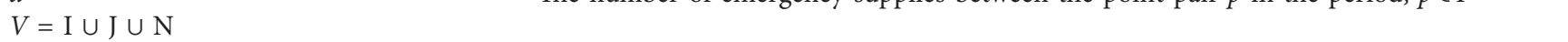 } \\
\hline$H$ & The opening cost of the fourth-party logistics service \\
\hline$C_{I}$ & The information transaction cost of the traditional bulk cargo transport network \\
\hline$E$ & Carbon emissions from bulk cargo transport networks \\
\hline$Q_{n}$ & Maximum load of carbon emission capacity of all vehicles in the freight enterprise $n$ \\
\hline$x_{i j k n} \in\{0,1\}$ & $\begin{array}{l}\text { If the vehicle } \mathrm{k} \text { is transported from the shipper } j \text { to the consignee } \mathrm{i} \text { via the freight enterprise } n \text {, then } \\
\qquad x_{i j k n}=1 ; \text { otherwise, } x_{i j k n}=0\end{array}$ \\
\hline$y_{n} \in\{0,1\}$ & If the fourth-party logistics service is opened, then $y_{n}=1$; otherwise, $y n=0$ \\
\hline$a_{i j k n}^{p} \in\{0,1\}$ & $\begin{array}{r}\text { If the shipper } j \text { to the consignee } i \text { is transported by the vehicle } k \text { and serves the point pair } p \text { via freight } \\
\text { enterprise } n \text {, then } a_{p i j k n}=1 \text {; otherwise, } a_{p i i k n}=0\end{array}$ \\
\hline$b_{n}^{p} \in\{0,1\}$ & If the freight enterprise $n$ is served the supply and demand point $p$, then $b_{n}^{p}=1$; otherwise, $b_{n}^{p}=0$. \\
\hline $\begin{array}{l}X^{+}=\left\{x_{i j k n} \mid \forall i \in V, \forall j \in V, \forall n \in V\right. \\
\forall k \in K\}\end{array}$ & \\
\hline$X^{-}=\left\{a_{p i j k n} \mid \forall i \in \mathrm{V}, \forall j \in V, \forall n \in V\right.$ & \\
\hline$\forall k \in K, \forall p \in P\}$ & \\
\hline$Y=\left\{y_{n} \mid \forall n \in N\right\}$ & \\
\hline
\end{tabular}

$$
\begin{gathered}
q_{i j k n} \geq 0, \quad \forall i \in V, \forall j \in V, \forall k \in K, \\
\sum_{j \in J} q_{i j k n} \leq S_{i}, \quad \forall i \in I, \\
\sum_{i \in I} q_{i j k n} \leq D_{j}, \quad \forall j \in J \\
\sum_{p \in P} b_{n}^{p} q_{i j k n} \leq W_{n} y_{n}, \quad \forall n \in N .
\end{gathered}
$$

Constraint (9) is the carbon emission constraint of freight enterprises. Constraint (10) is a transport route capacity constraint of freight enterprise $n$. Constraint (11) aims to determine the carbon emissions of freight enterprises. Constraint (12) ensures that the volume of freight sent by all shippers is the same as the consignee receives. Constraints (13)-(15) indicate the capacity constraints for transport between points. Constraint (16) represents the capacity constraint on the bulk cargo of freight enterprise $n$.

$$
\begin{aligned}
& a_{i j k n}^{p} \in\{0,1\}, \quad \forall i \in V, \forall j \in V, \forall n \in V, \forall k \in K, \forall p \in P, \\
& b_{n}^{p} \in\{0,1\}, \quad \forall n \in N, \forall p \in P, \\
& x_{i j k n} \in\{0,1\}, \quad \forall i \in V, \forall j \in V, \forall n \in V, \forall k \in K, \\
& y_{n} \in\{0,1\}, \quad \forall n \in N .
\end{aligned}
$$

Constraints (17)-(20) are $0-1$ decision variables.

3.4. Algorithm Design. Optimizing the green transport network for bulk cargoes of fourth-party logistics is an extension of the traditional logistics transport problem. In solving the minimum cost of the logistics network, due to the involvement of various shippers, freight enterprises, and locations of shippers, it is also an NP problem. This paper involves multiple shippers and multiple consignees. The calculation scale is large, and the solution is complicated. 
The corresponding intelligent optimization algorithm needs to be selected to solve the problem. Therefore, this paper improves the particle swarm optimization (PSO) algorithm to optimize the green transport of bulk cargoes based on fourth-party logistics.

3.4.1. Particle Swarm Optimization Algorithm. Particle swarm optimization (PSO) algorithm is an effective global optimization algorithm derived from the study of bird predation behavior and is used to solve the optimization problem. Similar to other evolutionary algorithms, PSO also searches for optimal solutions in complex spaces through cooperation and competition among individuals. PSO has the characteristics of evolutionary computing and swarms intelligence. It is an optimization algorithm based on swarm intelligence theory, which guides the optimization search through swarm intelligence generated by the competition and cooperation among particles in the swarm. PSO retains a population-based global search strategy compared with traditional evolutionary algorithms, but its "speed-displacement" model is simple to operate and avoids complex genetic operations. Its unique memory enables it to dynamically track the current search situation to adjust the search strategy.

In PSO, each optimization problem's solution is considered a bird in the search space, that is, "particle." First, as the initial population is generated, a group of particles is randomly initialized in a feasible solution space. Each particle is a feasible solution, and an objective function evaluates its fitness value. Each particle moves in the solution space, and its flight direction and distance are determined by velocity. Usually, the particle follows the current optimal particle to search in the solution space. During each iteration, the particle will track two "extreme values" to update itself. One is the optimal solution found by itself, and the other is the optimal solution currently found by the whole population. This extremum is the optimal global solution.

This paper assumes that the vehicle $k$ transported between shipper $j$ and consignee $i$ refers to particles. These particles are searched in the $p$-dimensional space. The swarm consisting of $P$ particles is $X=\left\{X_{1}, X_{2}, \ldots, X_{P}\right\}$, where the position of each particle $X_{k}=\left\{X_{k 1}, X_{k 2}, \ldots, X_{k P}\right\}$ represents a feasible solution to the optimization problem. Each particle has a velocity, which is $V_{k}=\left\{V_{k 1}, V_{k 2}, \ldots\right.$, $\left.V_{k P}\right\}$. Particles search for new solutions by constantly adjusting their positions, and each particle can remember the optimal solution it has searched for. In each iteration, the velocity and position of the particle are constantly updated according to the optimal historical position of the individual particle $L_{k}=\left\{L_{k 1}, L_{k 2}, \ldots, L_{k P}\right\}^{T}$ and the global optimal position $L_{g}=\left\{L_{g 1}, L_{g 2}, \ldots, L_{g P}\right\}^{T}$ to achieve population purification. The formula for updating the velocity and position of the $k$ th $p$-dimensional particle is as follows:
$V_{k p}^{z+1}=\omega \times V_{k p}^{z}+c_{1} \times r_{1} \times\left(P_{k p}^{z}-X_{k p}^{z}\right)+c_{2} \times r_{2} \times\left(P_{k p}^{z}-X_{k p}^{z}\right)$

$X_{k p}^{z+1}=X_{k p}^{z}+V_{k p}^{z+1}$.

In formulas (21) and (22), $z$ represents the current number of iterations; $c_{1}, c_{1}$ represent the acceleration factor; $r_{1}, r_{2}$ represent the random number between $[0,1]$; and $\omega$ is the inertia weight, and its size has a great effect on the convergence of PSO.

\subsubsection{Improved Implementation of Particle Swarm Optimi-} zation Algorithm. This paper designs a constraint processing mechanism based on the traditional PSO algorithm that allocates the region and location of the solution by Pareto dominance. It then searches through the feasible region by the PSO algorithm. After several iterative evolutions, the infeasible solution is finally eliminated, and the optimal solution is retained. According to the well-defined particle swarm structure, this paper adopts an improved particle swarm optimization algorithm (IPSO) to optimize the many-to-many transport cost of the fourth-party logistics network under low-carbon emissions.

Step 1. Initialize all parameters such as $V_{k}, L_{k}$, and $L_{g}$ in the particle swarm and generate the initial Pareto solution set with $V_{k}, L_{k}$, and $L_{g}$.

Step 2. Calculate fitness with $V_{k}, L_{k}$, and $L_{g}$.

Step 3. According to the Pareto solution set of different shippers and consignees, update $V_{k}, L_{k}$, and $L_{g}$.

Step 4. Correct the position and velocity of the particles beyond the boundary. For each particle $k$, compare its fitness value with the best position $L_{k}$ in the Pareto solution set. If it is better, update $L_{k}$. The update of $L_{g}$ is the same as $L_{k}$.

Step 5. The algorithm is ended if the end condition is reached (get a sufficiently good position or the maximum number of iterations) [20]. The optimal global solution is output; otherwise, continue the iteration.

The pseudocode of the IPSO algorithm is shown in Algorithm 1.

\subsubsection{Design of Constraint Processing Mechanism.} Constraints are an essential part of many-to-many logistics network optimization problems. The key to using the IPSO algorithm to solve the many-to-many optimization problem lies in designing effective constraint processing mechanisms. Because there are many constraints involved, this paper designs a flow allocation algorithm, a transportation capacity limit conversion algorithm, and a capacity adjustment algorithm to solve the mixed-integer programming model between multiple shippers and multiple consignees under constraints. 


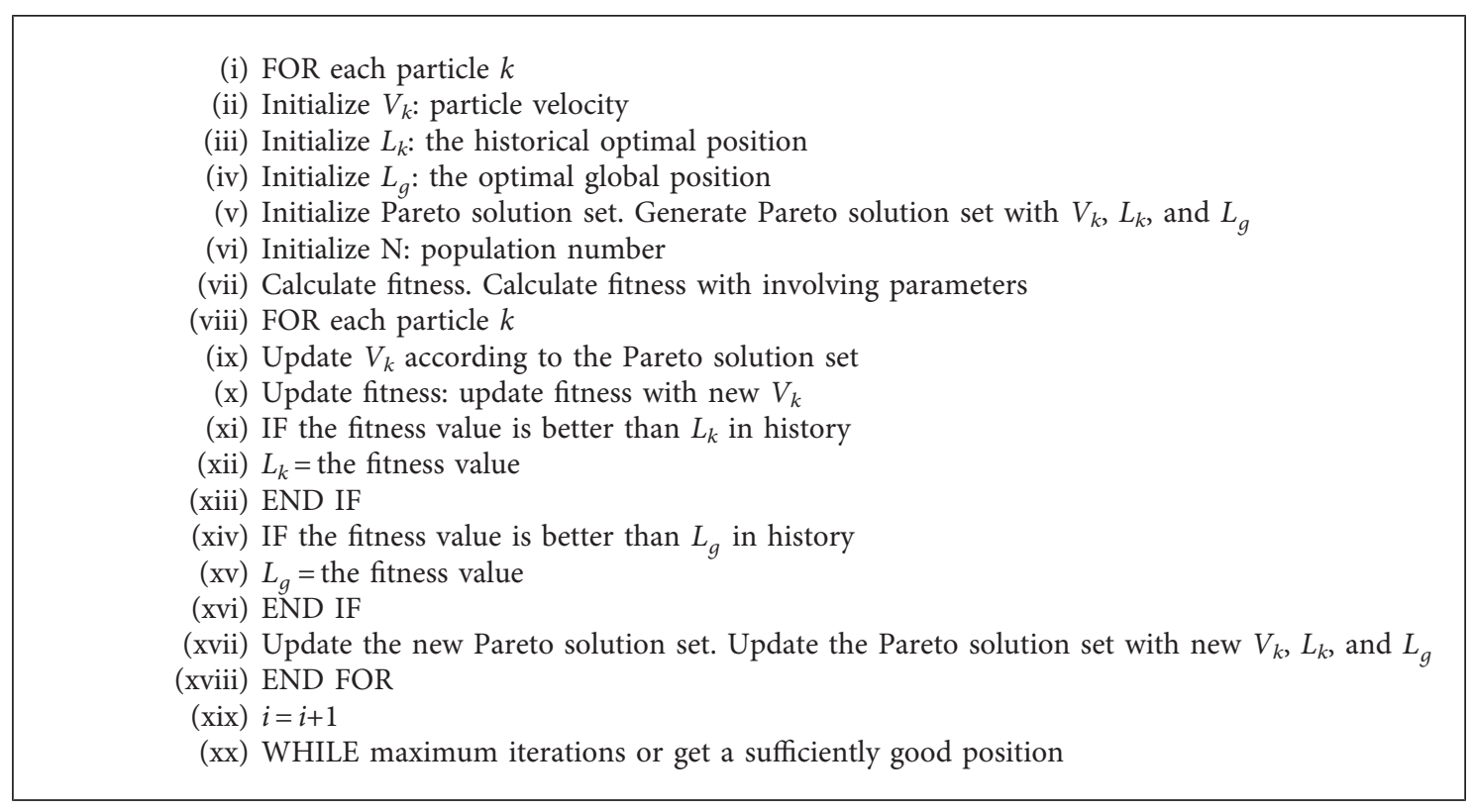

Algorithm 1: The improved particle swarm optimization algorithm.

In order to determine the cargo flow between the various facilities, this paper converts formulas (3)-(8), (11), and (12) into algorithmic languages for expression under the condition of solving objective functions (1) and (2) to select the method with the lowest integrated cost and carbon emission among the various nodes for bulk cargo transport. In order to ensure that the transportation volume between nodes does not exceed its carrying capacity, this paper uses a penalty function to convert transportation capacity constraints (9) and (10) to ensure that the bulk cargo volume of each transportation mode does not exceed its corresponding capacity limit. In order to make the opened nodes operate within their capabilities, this paper converts capacity constraints (13)-(16) into MATLAB language for expression, thus selecting the node with the lowest integrated cost for opening.

\section{Experiments and Data Analysis}

In order to optimize the fourth-party green transport network mode of bulk cargoes, this paper uses IPSO to test the fourth-party green transport network of bulk cargoes with three different conditions: large, medium, and small. The models and algorithms are coded in MATLAB language, and experiments are performed on different computers. The nodes and data involved in this experimental calculation are mainly based on Li et al.'s improved particle swarm optimization algorithm and reasonable adjustment and optimizations [20].

Assume that in the fourth-party logistics mode, different freight enterprises undertake bulk cargo transport tasks from multiple shippers $j$ to consignee $i$. The carbon emission factor per unit product unit distance in road transport $\beta_{1}$ is $0.056(\mathrm{~kg} / \mathrm{t} \cdot \mathrm{km})$, and the carbon emission factor per unit product unit distance in railway transport $\beta_{2}$ is $0.017(\mathrm{~kg} / \mathrm{t} \cdot \mathrm{km})$ [20]. The carbon tax rate of transport networks with fourth-party logistics service $\alpha_{1}$ is 250 yuan/ $t$, and that of the transport network without fourthparty logistics service $\alpha$ is 300 yuan/t [31]. The cost of loading and unloading the bulk cargo during the road-torail transport is 1000 yuan (combined with the opening cost of the rail transport service when calculating), and each transport process includes two times of loading and unloading. In the bulk cargo logistics network assumed in this paper, because the front-end transport and the terminal transport part of the rail or road transport process use road transportation, the costs incurred are the same, that is, only the cost of railway transport in the mainline transport, the sum of the loading and unloading costs at both ends, and the road transport integrated cost are considered to be compared in the calculation of the integrated cost. For simplicity, this paper does not consider the fixed construction costs of shippers, consignees, and freight enterprises.

\subsection{Green Transport Optimization for the Small Bulk Cargo} Transport Network. A small bulk cargo network consists of 2 shippers, 2 freight enterprises, and 2 consignees. After receiving the bulk cargo provided by the shipper, the freight enterprises in the network can choose two main transport modes, road and rail transport, to transfer the bulk cargo to the consignee, and the shipper has three vehicles under each transport mode to perform the delivery task. The related operation and management data of each node of the logistics network are shown in Table 2.

The shipper contracts bulk cargo transport to the corresponding freight enterprises and chooses the road and rail transport modes to transport the cargo to the consignee according to the actual situation. The distances between the nodes are shown in Table 3. 
TABLE 2: Parameter of the small bulk cargo transport network.

\begin{tabular}{|c|c|c|c|c|c|}
\hline Parameter description & Parameter & Value & Parameter description & Parameter & Value \\
\hline Transport capacity of each vehicle & $Q_{k}$ & $1000 \mathrm{t}$ & $\begin{array}{l}\text { Number of vehicles in each } \\
\text { freight enterprise }\end{array}$ & K & 3 \\
\hline Transport capacity of freight enterprise 1 & $G_{1}$ & $1800 \mathrm{t}$ & Cargo required by consignee 1 & $S_{1}$ & $800 t$ \\
\hline Transport capacity of freight enterprise 2 & $G_{2}$ & $1900 \mathrm{t}$ & Cargo required by consignee 2 & $S_{2}$ & $1000 \mathrm{t}$ \\
\hline Freight volume from shipper 1 & $D_{1}$ & $3000 \mathrm{t}$ & Freight volume from shipper 2 & $\mathrm{D}_{2}$ & $2850 \mathrm{t}$ \\
\hline Maximum load of road freight enterprise & $W_{1}$ & $2000 \mathrm{t}$ & $\begin{array}{c}\text { Rail handing capacity of freight } \\
\text { enterprise } 1\end{array}$ & $W_{1}^{\prime}$ & $2500 t$ \\
\hline Maximum load of rail freight enterprise & $W_{2}$ & $2100 \mathrm{t}$ & $\begin{array}{c}\text { Rail handing capacity of freight } \\
\text { enterprise } 2\end{array}$ & $W_{2}^{\prime}$ & $2600 t$ \\
\hline $\begin{array}{l}\text { Information transaction cost of traditional } \\
\text { transportation network }\end{array}$ & $C_{I}$ & 3000 yuan & $\begin{array}{l}\text { Opening cost of fourth-party } \\
\text { logistics service }\end{array}$ & $H$ & 30000 yuan \\
\hline Unit road transport costs of enterprises & $c_{i j k n}$ & $\begin{array}{l}165.93 \text { yuan } / \\
t \times \mathrm{km}\end{array}$ & $\begin{array}{l}\text { Unit rail transport costs of } \\
\text { enterprises }\end{array}$ & $c_{i j k n}$ & $\begin{array}{l}110.62 \text { yuan } / \\
t \times \mathrm{km}\end{array}$ \\
\hline
\end{tabular}

TABLe 3: The distance between nodes in the small bulk cargo transport network $(\mathrm{km})$.

\begin{tabular}{lcc}
\hline & Freight enterprise 1 & Freight enterprise 2 \\
\hline Shipper 1 & 140 & 190 \\
Shipper 2 & 210 & 175 \\
Consignee 1 & 325 & 320 \\
Consignee 2 & 350 & 320 \\
\hline
\end{tabular}

TABLE 4: Circulation of cargoes at supply and demand points in the small bulk cargo transport network ( 0 means there is no supply and demand relationship).

\begin{tabular}{lcc}
\hline & Consignee 1 & Consignee 2 \\
\hline Shipper 1 & 0 & 600 \\
Shipper 2 & 500 & 0 \\
\hline
\end{tabular}

Combining the above data, this paper assumes that the supply and demand of each shipper and consignee and the circulation of bulk cargoes are as shown in Table 4 .

After the MATLAB evolution algorithm, the integrated cost of road and railway transport in the condition of the small bulk cargo transport network in a limited time is obtained, as shown in Figure 3. In the condition of the small bulk cargo transport network, because the number of shippers and consignees is less than the number of vehicles held by freight enterprises, the logistics network transport route is not unique. When the fourth-party logistics service is not selected, the integrated cost of the road transport network is 187,210 yuan, and the integrated cost of the railway transport network is 127,330 yuan; when selecting the fourth-party logistics service, the integrated cost of the road transport network is 198,700 yuan, and the integrated cost of the railway transport network is 139,230 yuan. Besides, in the small bulk cargo transport network, the carbon emissions from road transport are 10.2 tons, and the carbon emissions from railway transport are 2.0 tons. Promoting the transfer of transport methods from road to railway can reduce carbon emissions by $80.39 \%$.

\subsection{Green Transport Optimization for the Medium Bulk Cargo} Transport Network. A medium bulk cargo network consists of 3 shippers, 3 freight enterprises, and 4 consignees. After receiving the bulk cargo provided by the shipper, the freight enterprise in the network can choose two main transport modes, road and rail transport, to transfer the bulk cargo to the consignee. The shipper has three vehicles under each transport mode to perform the delivery task. The related operation and management data of each node of the logistics network are shown in Table 5 .

The shipper contracts bulk cargo transport to the corresponding freight enterprises and chooses the road and rail transport modes to transport the cargo to the consignee according to the actual situation. The distances between the nodes are shown in Table 6.

Combining the above data, this paper assumes that the supply and demand of each shipper and consignee and the circulation of bulk cargoes are as shown in Table 7 .

After the MATLAB evolution algorithm, the integrated cost of road and railway transport of the medium bulk cargo transport network in a limited time is shown in Figure 4. When the fourth-party logistics service is not selected, the integrated cost of the road transport network is 540,260 yuan, and the integrated cost of the railway transport network is 366,820 yuan; when selecting the fourth-party logistics service, the integrated cost of the road transport network is 546,800 yuan, and the integrated cost of the railway transport network is 374,520 yuan. Besides, in the medium bulk cargo transport network, the carbon emissions from road transport are 29.2 tons, and the carbon emissions from railway transport are 6.0 tons. Promoting the transfer of transport methods from road to railway can reduce carbon emissions by $79.45 \%$.

Table 8 shows the route flow of different consignees receiving the products sent by the shipper in the form of road and rail transport. Taking consignee 1 as an example, when choosing road transport, the cargoes received are transported by shipper 2 via the third freight vehicle of 


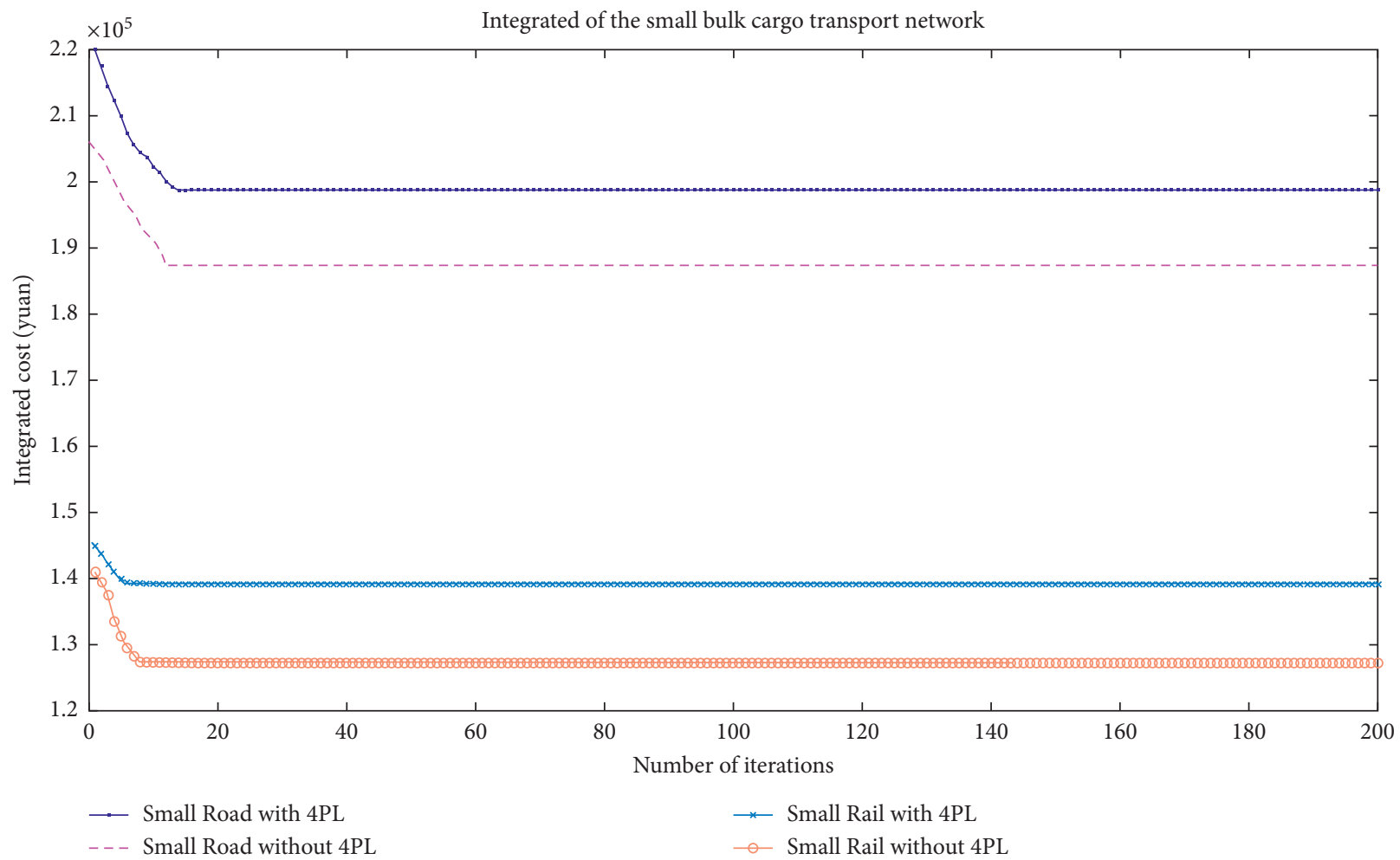

FIGURE 3: The integrated cost of the small bulk cargo transport network.

TABle 5: Parameter of the medium bulk cargo transport network.

\begin{tabular}{|c|c|c|c|c|c|}
\hline Parameter description & Parameter & Value & Parameter description & Parameter & Value \\
\hline Transport capacity of each vehicle & $Q_{k}$ & $1000 \mathrm{t}$ & $\begin{array}{l}\text { Number of vehicles in each } \\
\text { freight enterprise }\end{array}$ & $K$ & 3 \\
\hline Transport capacity of freight enterprise 1 & $G_{1}$ & $1800 \mathrm{t}$ & Cargo required by consignee 1 & $S_{1}$ & $800 \mathrm{t}$ \\
\hline Transport capacity of freight enterprise 2 & $G_{2}$ & $1900 \mathrm{t}$ & Cargo required by consignee 2 & $S_{2}$ & $1000 t$ \\
\hline Transport capacity of freight enterprise 3 & $G_{3}$ & $2000 \mathrm{t}$ & Cargo required by consignee 3 & $S_{3}$ & $650 \mathrm{t}$ \\
\hline Freight volume from shipper 1 & $D_{1}$ & $3000 \mathrm{t}$ & Cargo required by consignee 4 & $S_{4}$ & $700 \mathrm{t}$ \\
\hline Freight volume from shipper 2 & $D_{2}$ & $2850 \mathrm{t}$ & Freight volume from shipper 3 & $D_{3}$ & $3500 t$ \\
\hline Road handing capacity of freight enterprise 1 & $W_{1}$ & $2000 \mathrm{t}$ & $\begin{array}{c}\text { Rail handing capacity of freight } \\
\text { enterprise } 1\end{array}$ & $W_{1}^{\prime}$ & $2500 t$ \\
\hline Road handing capacity of freight enterprise 2 & $W_{2}$ & $2100 \mathrm{t}$ & $\begin{array}{c}\text { Rail handing capacity of freight } \\
\text { enterprise } 2\end{array}$ & $W_{2}^{\prime}$ & $2600 t$ \\
\hline Road handing capacity of freight enterprise 3 & $W_{3}$ & $2200 t$ & $\begin{array}{c}\text { Rail handing capacity of freight } \\
\text { enterprise } 3\end{array}$ & $W_{3}^{\prime}$ & $2600 t$ \\
\hline $\begin{array}{l}\text { Information transaction cost of traditional } \\
\text { transportation network }\end{array}$ & $C_{I}$ & 6000 yuan & $\begin{array}{l}\text { Opening cost of fourth-party } \\
\text { logistics service }\end{array}$ & $H$ & 30000 yuan \\
\hline Unit road transport costs of enterprises & $c_{i j k n}$ & $\begin{array}{l}165.93 \mathrm{yuan} / t \\
\times \mathrm{km}\end{array}$ & $\begin{array}{l}\text { Unit rail transport costs of } \\
\text { enterprises }\end{array}$ & $c_{i j k n}^{\prime}$ & $\begin{array}{l}110.62 \mathrm{yuan} / t \\
\times \mathrm{km}\end{array}$ \\
\hline
\end{tabular}

TABLE 6: The distance between nodes in the medium bulk cargo transport network $(\mathrm{km})$.

\begin{tabular}{lccc}
\hline & Freight enterprise 1 & Freight enterprise 2 & Freight enterprise 3 \\
\hline Shipper 1 & 140 & 190 & 65 \\
Shipper 2 & 210 & 175 & 125 \\
Shipper 3 & 190 & 240 & 105 \\
Consignee 1 & 325 & 320 & 370 \\
Consignee 2 & 350 & 320 & 335 \\
Consignee 3 & 345 & 320 & 340 \\
Consignee 4 & 355 & 320 & 365 \\
\hline
\end{tabular}


TABLE 7: Circulation of cargoes at supply and demand points in the medium bulk cargo transport network.

\begin{tabular}{lcccc}
\hline & Consignee 1 & Consignee 2 & Consignee 3 & Consignee 4 \\
\hline Shipper 1 & 0 & 600 & 0 & 400 \\
Shipper 2 & 500 & 0 & 200 & 300 \\
Shipper 3 & 300 & 400 & 450 & 0 \\
\hline
\end{tabular}

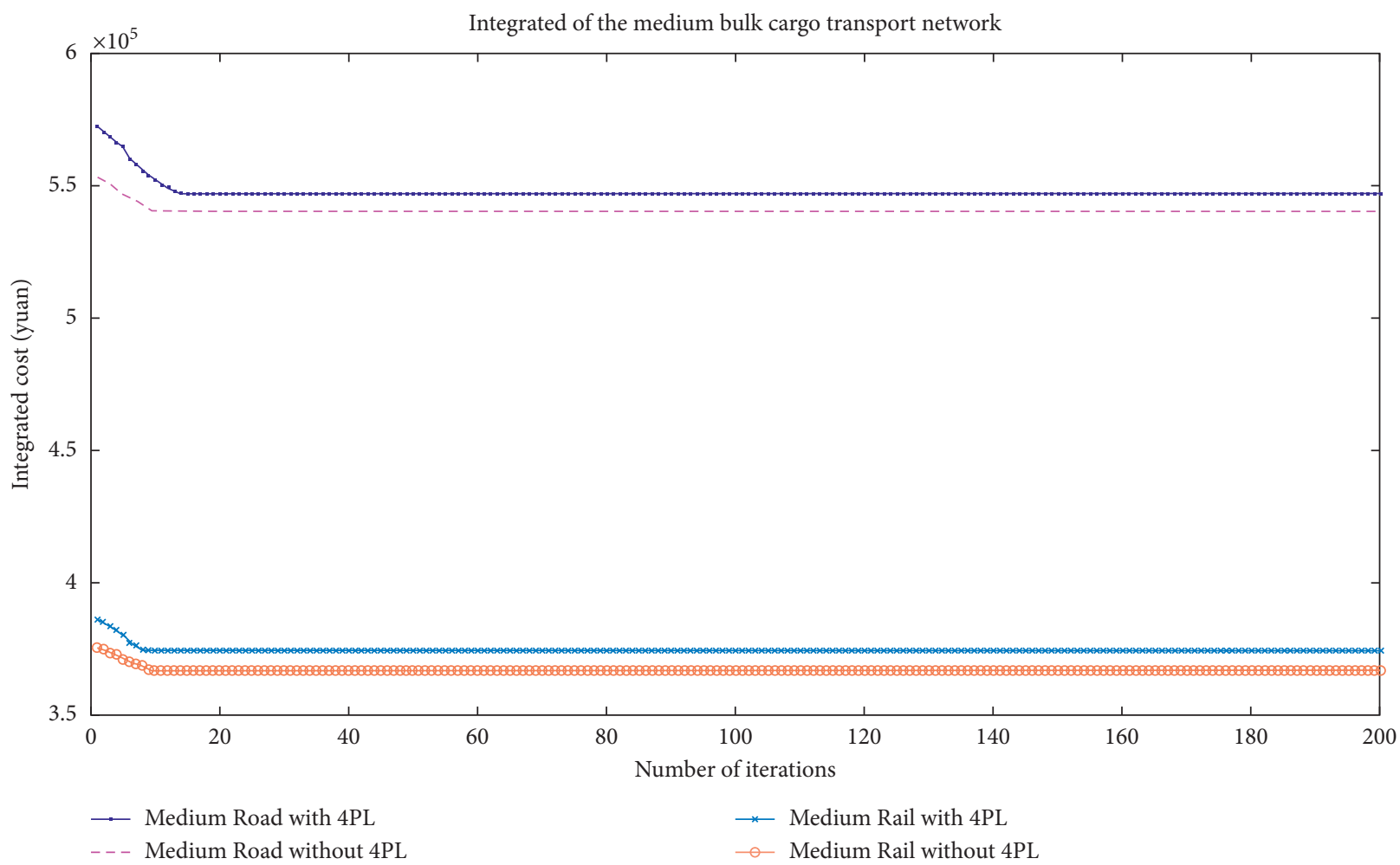

FIGURE 4: The integrated cost of the medium bulk cargo transport network.

TABLE 8: The flow of supply and demand points in the medium bulk cargo transport network.

\begin{tabular}{lccc}
\hline \multicolumn{2}{c}{ Road transport route } & \multicolumn{2}{c}{ Rail transport route } \\
Consignee & Route flow & Consignee & Route flow \\
\hline 1 & $2 \longrightarrow 2 \longrightarrow 1(3)$ & 1 & $2 \longrightarrow 3 \longrightarrow 1(3)$ \\
1 & $3 \longrightarrow 2 \longrightarrow 1(3)$ & 1 & $3 \longrightarrow 3 \longrightarrow 1(3)$ \\
2 & $1 \longrightarrow 2 \longrightarrow 2(2)$ & 2 & $1 \longrightarrow 3 \longrightarrow 2(1)$ \\
2 & $3 \longrightarrow 3 \longrightarrow 2(2)$ & 2 & $3 \longrightarrow 3 \longrightarrow 2(3)$ \\
3 & $2 \longrightarrow 3 \longrightarrow 3(2)$ & 3 & $2 \longrightarrow 2 \longrightarrow 3(2)$ \\
3 & $3 \longrightarrow 3 \longrightarrow 3(1)$ & 3 & $3 \longrightarrow 3 \longrightarrow 3(1)$ \\
4 & $1 \longrightarrow 2 \longrightarrow 4(2)$ & 4 & $1 \longrightarrow 2 \longrightarrow 4(3)$ \\
4 & $2 \longrightarrow 2 \longrightarrow 4(2)$ & 4 & $2 \longrightarrow 2 \longrightarrow 4(1)$ \\
\hline
\end{tabular}

freight enterprise 2 and by shipper 3 via the first freight vehicle of freight enterprise 2. In the selection of rail transport, the cargoes received by shipper 2 are those transported by shipper 2 via the first freight vehicle of freight enterprise 3 and by shipper 3 via the third freight vehicle of freight enterprise 3.

4.3. Green Transport Optimization for the Large Bulk Cargo Transport Network. A large bulk cargo network consists of 5 shippers, 5 freight enterprises, and 6 consignees. After receiving the bulk cargo provided by the shipper, the freight enterprise in the network can choose two main transport modes, road and rail transport, to transfer the bulk cargo to the consignee, and the shipper has three vehicles under each transport mode to perform the delivery task. The related operation and management data of each node of the logistics network are shown in Table 9.

The shipper contracts bulk cargo transport to the corresponding freight enterprises and chooses the road and rail transport modes to transport the cargo to the consignee according to the actual situation. The distances between the nodes are shown in Table 10.

Combining the above data, this paper assumes that the supply and demand of each shipper and consignee and the circulation of bulk cargoes are as shown in Table 11.

After the MATLAB evolution algorithm, the integrated cost of road and railway transport of the large bulk cargo transport network in a limited time is shown in Figure 5. When the fourth-party logistics service is not selected, the integrated cost of the road transport network is $1,226,300$ yuan, and the integrated cost of the railway transport network is 824,180 yuan. When selecting the fourth-party logistics service, the integrated cost of the road transport network is 1,203,100 yuan, and the integrated cost of the 
TABLe 9: Parameter of the large bulk cargo transport network.

\begin{tabular}{|c|c|c|c|c|c|}
\hline Parameter description & Parameter & Value & Parameter description & Parameter & Value \\
\hline Transport capacity of each vehicle & $Q_{k}$ & $1000 \mathrm{t}$ & $\begin{array}{l}\text { Number of vehicles in each } \\
\text { freight enterprise }\end{array}$ & $K$ & 3 \\
\hline Transport capacity of freight enterprise 1 & $G_{1}$ & $1800 \mathrm{t}$ & Cargo required by consignee 1 & $S_{1}$ & $800 \mathrm{t}$ \\
\hline Transport capacity of freight enterprise 2 & $G_{2}$ & $1900 t$ & Cargo required by consignee 2 & $S_{2}$ & $1000 t$ \\
\hline Transport capacity of freight enterprise 3 & $G_{3}$ & $2000 \mathrm{t}$ & Cargo required by consignee 3 & $S_{3}$ & $650 \mathrm{t}$ \\
\hline Transport capacity of freight enterprise 4 & $G_{4}$ & $2100 \mathrm{t}$ & Cargo required by consignee 4 & $S_{4}$ & $700 t$ \\
\hline Transport capacity of freight enterprise 5 & $G_{5}$ & $1700 t$ & Cargo required by consignee 5 & $S_{5}$ & $1100 t$ \\
\hline Freight volume from shipper 1 & $D_{1}$ & $3000 t$ & Cargo required by consignee 6 & $S_{6}$ & $1000 t$ \\
\hline Freight volume from shipper 2 & $D_{2}$ & $2850 \mathrm{t}$ & Freight volume from shipper 4 & $D_{4}$ & $3200 \mathrm{t}$ \\
\hline Freight volume from shipper 3 & $D_{3}$ & $3500 \mathrm{t}$ & Freight volume from shipper 5 & $D_{5}$ & $2700 \mathrm{t}$ \\
\hline Road handing capacity of freight enterprise 1 & $W_{1}$ & $2000 \mathrm{t}$ & $\begin{array}{c}\text { Rail handing capacity of freight } \\
\text { enterprise } 1\end{array}$ & $W_{1}^{\prime}$ & $2500 t$ \\
\hline Road handing capacity of freight enterprise 2 & $W_{2}$ & $2100 \mathrm{t}$ & $\begin{array}{c}\text { Rail handing capacity of freight } \\
\text { enterprise } 2\end{array}$ & $W_{2}^{\prime}$ & $2600 t$ \\
\hline Road handing capacity of freight enterprise 3 & $W_{3}$ & $2200 t$ & $\begin{array}{c}\text { Rail handing capacity of freight } \\
\text { enterprise } 3\end{array}$ & $W_{3}^{\prime}$ & $2600 t$ \\
\hline Road handing capacity of freight enterprise 4 & $W_{4}$ & $2200 \mathrm{t}$ & $\begin{array}{c}\text { Rail handing capacity of freight } \\
\text { enterprise } 4\end{array}$ & $W_{4}^{\prime}$ & $2700 \mathrm{t}$ \\
\hline Road handing capacity of freight enterprise 5 & $W_{5}$ & $1800 \mathrm{t}$ & $\begin{array}{c}\text { Rail handing capacity of freight } \\
\text { enterprise } 5\end{array}$ & $W_{5}^{\prime}$ & $2400 \mathrm{t}$ \\
\hline $\begin{array}{l}\text { Information transaction cost of traditional } \\
\text { transportation network }\end{array}$ & $C_{I}$ & 11000 yuan & $\begin{array}{l}\text { Opening cost of fourth-party } \\
\text { logistics service }\end{array}$ & $H$ & 30000 yuan \\
\hline Unit road transport costs of enterprises & $c_{i j k n}$ & $\begin{array}{l}165.93 \mathrm{yuan} / t \\
\times \mathrm{km}\end{array}$ & $\begin{array}{l}\text { Unit rail transport costs of } \\
\text { enterprises }\end{array}$ & $c_{i j k n}^{\prime}$ & $\begin{array}{l}110.62 \mathrm{yuan} / t \\
\times \mathrm{km}\end{array}$ \\
\hline
\end{tabular}

TABLE 10: The distance between nodes in the large bulk cargo transport network $(\mathrm{km})$.

\begin{tabular}{|c|c|c|c|c|c|}
\hline & Freight enterprise 1 & Freight enterprise 2 & Freight enterprise 3 & Freight enterprise 4 & Freight enterprise 5 \\
\hline Shipper 1 & 140 & 190 & 65 & 220 & 90 \\
\hline Shipper 2 & 210 & 175 & 125 & 150 & 185 \\
\hline Shipper 3 & 190 & 240 & 105 & 70 & 165 \\
\hline Shipper 4 & 145 & 165 & 90 & 95 & 180 \\
\hline Shipper 5 & 155 & 75 & 130 & 100 & 195 \\
\hline Consignee 1 & 325 & 320 & 370 & 310 & 370 \\
\hline Consignee 2 & 350 & 320 & 335 & 315 & 375 \\
\hline Consignee 3 & 345 & 320 & 340 & 330 & 370 \\
\hline Consignee 4 & 355 & 320 & 365 & 325 & 385 \\
\hline Consignee 5 & 330 & 320 & 380 & 305 & 390 \\
\hline Consignee 6 & 300 & 320 & 365 & 300 & 395 \\
\hline
\end{tabular}

railway transport network is 808,520 yuan. Besides, in the medium bulk cargo transport network, the carbon emissions from road transport are 64.0 tons, and the carbon emissions from railway transport are 13.2 tons. Promoting the transfer of transport methods from road to railway can reduce carbon emissions by $79.38 \%$.

Table 12 shows the route flow of different consignees receiving the products sent by the shipper in the form of road and rail transport.

4.4. Algorithm Performance Analysis. The algorithm is implemented using MATLAB R2018a programming. In order to verify the algorithm performance, this paper defines the relevant performance parameters of the algorithm. The algorithm runs 100 times, BEST represents the best solution of $100, \mathrm{BAD}$ the worst solution, MEAN represents the average value, and TIME represents the average time consumed by the algorithm once. We solve large, medium, and small rail transport networks using traditional PSO and IPSO, respectively. The results of the algorithm comparison are shown in Table 13.

It shows that both PSO and IPSO can more effectively solve the problems for the three cases of different scales. For small logistics networks, both algorithms obtain better solutions quickly. However, with the increase of the logistics network, the IPSO has gradually shown higher stability than the traditional PSO. By comparing the logistics networks of three scales, it can be found that IPSO consumes less time and has higher stability of the solution than PSO. It means 
TABLE 11: Circulation of cargoes at supply and demand points in the large bulk cargo transport network.

\begin{tabular}{|c|c|c|c|c|c|c|}
\hline Algorithm & Consignee 1 & Consignee 2 & Consignee 3 & Consignee 4 & Consignee 5 & Consignee 6 \\
\hline Shipper 1 & 0 & 600 & 0 & 400 & 0 & 300 \\
\hline Shipper 2 & 500 & 0 & 200 & 300 & 250 & 0 \\
\hline Shipper 3 & 300 & 400 & 450 & 0 & 0 & 400 \\
\hline Shipper 4 & 400 & 0 & 0 & 300 & 450 & 500 \\
\hline Shipper 5 & 0 & 0 & 350 & 450 & 500 & 0 \\
\hline
\end{tabular}

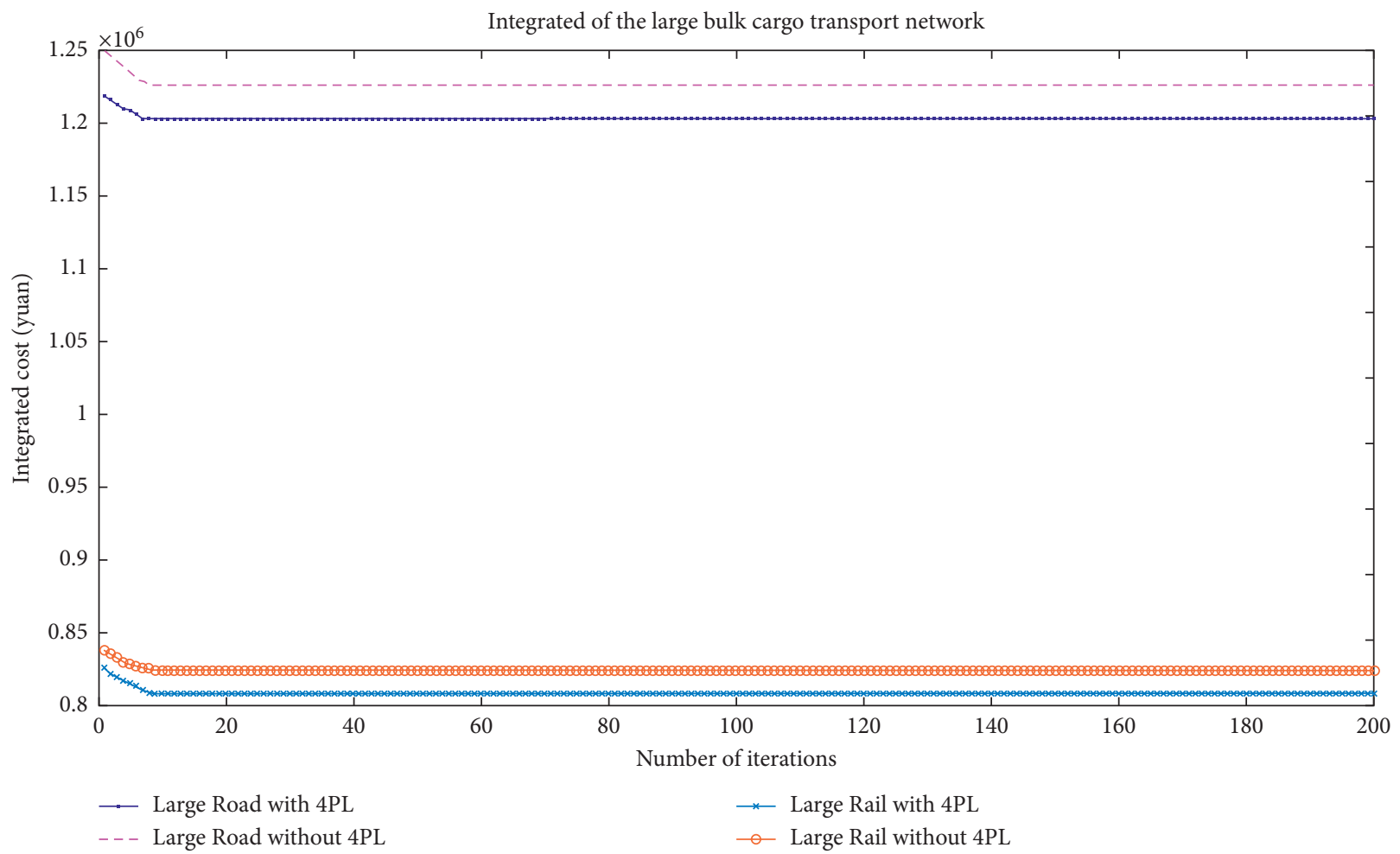

FIgURE 5: The integrated cost of the large bulk cargo transport network.

TABLE 12: The flow of supply and demand points in the large bulk cargo transport network.

\begin{tabular}{lccc}
\hline \multicolumn{2}{c}{ Road transport route } & \multicolumn{2}{c}{ Rail transport route } \\
Consignee & Route flow & Consignee & Route flow \\
\hline 1 & $2 \longrightarrow 1 \longrightarrow 1(2)$ & 1 & $2 \longrightarrow 2 \longrightarrow 1(1)$ \\
1 & $3 \longrightarrow 1 \longrightarrow 1(3)$ & 1 & $3 \longrightarrow 4 \longrightarrow 1(3)$ \\
1 & $4 \longrightarrow 2 \longrightarrow 1(3)$ & 1 & $4 \longrightarrow 1 \longrightarrow 1(2)$ \\
2 & $1 \longrightarrow 2 \longrightarrow 2(3)$ & 2 & $1 \longrightarrow 4 \longrightarrow 2(2)$ \\
2 & $3 \longrightarrow 4 \longrightarrow 2(1)$ & 2 & $3 \longrightarrow 1 \longrightarrow 2(3)$ \\
3 & $2 \longrightarrow 4 \longrightarrow 3(2)$ & 3 & $2 \longrightarrow 4 \longrightarrow 3(2)$ \\
3 & $3 \longrightarrow 2 \longrightarrow 3(3)$ & 3 & $3 \longrightarrow 2 \longrightarrow 3(1)$ \\
3 & $5 \longrightarrow 3 \longrightarrow 3(3)$ & 3 & $5 \longrightarrow 4 \longrightarrow 3(2)$ \\
4 & $1 \longrightarrow 4 \longrightarrow 4(2)$ & 4 & $1 \longrightarrow 2 \longrightarrow 4(3)$ \\
4 & $2 \longrightarrow 4 \longrightarrow 4(1)$ & 4 & $2 \longrightarrow 4 \longrightarrow 4(3)$ \\
4 & $4 \longrightarrow 2 \longrightarrow 4(2)$ & 4 & $4 \longrightarrow 1 \longrightarrow 4(1)$ \\
4 & $5 \longrightarrow 1 \longrightarrow 4(1)$ & 4 & $5 \longrightarrow 1 \longrightarrow 4(3)$ \\
5 & $2 \longrightarrow 4 \longrightarrow 5(2)$ & 5 & $2 \longrightarrow 2 \longrightarrow 5(1)$ \\
5 & $4 \longrightarrow 1 \longrightarrow 5(1)$ & 5 & $4 \longrightarrow 4 \longrightarrow 5(3)$ \\
5 & $5 \longrightarrow 3 \longrightarrow 5(1)$ & 5 & $5 \longrightarrow 1 \longrightarrow 5(1)$ \\
6 & $1 \longrightarrow 3 \longrightarrow 6(2)$ & 6 & $1 \longrightarrow 2 \longrightarrow 6(1)$ \\
6 & $3 \longrightarrow 4 \longrightarrow 6(1)$ & 6 & $3 \longrightarrow 2 \longrightarrow 6(2)$ \\
6 & $4 \longrightarrow 3 \longrightarrow 6(3)$ & 6 & $4 \longrightarrow 4 \longrightarrow 6(1)$ \\
\hline
\end{tabular}

that compared with PSO, IPSO can solve the proposed problem faster and more accurately, showing the improved method's effectiveness.

4.5. Comparison of Results. By comparing the costs of large, medium, and small bulk cargo logistics networks under road and rail transport conditions, this paper investigates the effect of low-carbon emission reduction effect of railway transport on road transport under different conditions of low-carbon emissions. The results are shown in Table 14.

From the above, in the small, medium, and large bulk cargo transport networks, the integrated cost of rail transport is lower than that of road transport, which plays the role of cost optimization. From the perspective of the benefits of the transport network, with the continuous expansion of the bulk cargo transport network's scale, the cost optimization rate of the transfer of bulk cargo from road transport to rail transport has gradually increased. From the perspective of the fourth-party logistics, when the bulk cargo transport network is small, the integrated cost of the transport network using fourth-party logistics is more than the integrated cost 
TABLE 13: PSO and IPSO performance in bulk cargo transport networks of different sizes.

\begin{tabular}{lccccc}
\hline Network scale & Algorithm & BEST & BAD & MEAN & TIME \\
\hline \multirow{2}{*}{ Small } & PSO & 127,330 & 127,330 & 127,330 & 127,330 \\
& IPSO & 127,330 & 127,330 & 366,880 & 7.2899 \\
\multirow{2}{*}{ Medium } & PSO & 366,820 & 372,820 & 366,820 & 13.0755 \\
\multirow{2}{*}{ Large } & IPSO & 366,820 & 366,820 & 824,960 & 12.2844 \\
& PSO & 824,180 & 837,180 & 21.2474 \\
& IPSO & 824,180 & 837,180 & 824,440 & 20.1656 \\
\hline
\end{tabular}

TABLE 14: Effect in bulk cargo transport networks of different sizes.

\begin{tabular}{|c|c|c|c|c|c|c|}
\hline $\begin{array}{l}\text { Network } \\
\text { scale }\end{array}$ & $\begin{array}{c}4 \mathrm{PL} \\
\text { service }\end{array}$ & $\begin{array}{l}\text { Transport } \\
\text { mode }\end{array}$ & $\begin{array}{l}\text { Integrated cost } \\
\text { (yuan) }\end{array}$ & $\begin{array}{c}\text { Cost optimization rate } \\
(\%)\end{array}$ & $\begin{array}{c}\text { Carbon emissions } \\
\text { (ton) }\end{array}$ & $\begin{array}{c}\text { Carbon emission optimization } \\
\text { rate (\%) }\end{array}$ \\
\hline \multirow{4}{*}{ Small } & \multirow{2}{*}{ No } & Road & 187210 & \multirow{2}{*}{31.96} & 1020 & \multirow{4}{*}{80.39} \\
\hline & & Railway & 127330 & & 200 & \\
\hline & \multirow{2}{*}{ Yes } & Road & 198700 & \multirow{2}{*}{29.93} & 510 & \\
\hline & & Railway & 139230 & & 100 & \\
\hline \multirow{4}{*}{ Medium } & \multirow{2}{*}{ No } & Road & 540260 & \multirow{2}{*}{32.10} & 2920 & \multirow{4}{*}{79.45} \\
\hline & & Railway & 366820 & & 600 & \\
\hline & \multirow{2}{*}{ Yes } & Road & 546800 & \multirow{2}{*}{31.51} & 1460 & \\
\hline & & Railway & 374520 & & 300 & \\
\hline \multirow{4}{*}{ Large } & \multirow{2}{*}{ No } & Road & 1226300 & \multirow{2}{*}{32.79} & 6400 & \multirow{4}{*}{79.38} \\
\hline & & Railway & 824180 & & 1320 & \\
\hline & \multirow{2}{*}{ Yes } & Road & 1203100 & \multirow{2}{*}{32.80} & 3200 & \\
\hline & & Railway & 808520 & & 660 & \\
\hline
\end{tabular}

of the transport network not using fourth-party logistics. With continuous expansion, the cost optimization benefits brought by fourth-party logistics gradually increase, surpassing the transport network that does not use fourth-party logistics. From the perspective of low-carbon emissions, in various transport networks with the same conditions, the carbon emissions generated by the implementation of railway transport are reduced by about $80 \%$ compared to the carbon emissions generated by road transport, which shows that the promotion of bulk cargoes from roads to railways transfer can play an essential role in reducing costs and emissions.

In summary, combining fourth-party logistics with green transport of bulk cargoes is more suitable for more extensive transport networks and transferring bulk cargo transport from road to railway. It is possible to significantly reduce the integrated cost and carbon emissions of bulk cargo transport networks. This shows that with the continuous expansion of the logistics network, the optimization benefits of rail transport for the optimization of integrated costs continue to increase, and the energy-saving and emission reduction effects are very remarkable. Using the fourth-party logistics mode to promote the "road to rail" of bulk cargo under low-carbon emission conditions can sufficiently reduce the integrated cost of the logistics network.

\section{Conclusions and Discussion}

At present, China's economy has changed from a highspeed growth stage to a high-quality development stage, and the people's attention to the sustainable development of the transportation industry has gradually increased. With the changes in national policies and the adjustment of the transport structure, railways should actively increase their transportation capacity to reduce carbon emissions from the transportation industry and promote green transport.

This paper discusses the optimization of bulk cargo green transport through fourth-party logistics. In the process of combining the rail transport of bulk cargoes with fourth-party logistics, this paper draws the following conclusions. (1) The fourth-party logistics guides the transfer of cargo from road transport to rail transport, which can effectively reduce carbon emissions and transport costs. (2) Fourth-party logistics is more suitable for large-scale bulk cargo transport networks and has lower benefits for small-scale bulk cargo transport networks. (3) The particle swarm optimization algorithm is improved. It can be found that IPSO consumes less time and has higher stability of the solution than PSO.

This paper mainly considers the transport mode selection, energy saving and emission reduction, and cost optimization benefits of the fourth-party logistics for the green transport of bulk cargoes. However, the optimization of the "first and last kilometre" of rail transport under the fourth-party logistics has not been further solved. In addition, this paper does not incorporate reverse logistics into the bulk cargo green transport network. In future research, this paper will further optimize the model and theory, on the one hand, to solve the "first and last kilometre" problem in the fourth-party logistics model of bulk 
cargo and, on the other hand, to explore the optimization of many-to-many reverse logistics under the fourth-party transport network by adding reverse logistics on the basis of the existing model.

\section{Data Availability}

The data that support the findings of this study are available from the corresponding author upon reasonable request.

\section{Conflicts of Interest}

The authors declare that they have no conflicts of interest.

\section{Acknowledgments}

The authors would like to express their gratitude to EditSprings (https://www.editsprings.com/) for expert linguistic services. The authors would also like to pay tribute to $\mathrm{Mr}$ Wang Xingpeng, Ms Zhang Pei, and Ms Zhang Qinghong, whose profound knowledge and effort helped in revising this paper. This research was funded by the Hebei Provincial Department of Education (grant no. SD 2021040) and supported by the Development Fund of Innovative Research Team of Shijiazhuang Tiedao University (grant no. SCT 202003).

\section{References}

[1] J. Wang, "The Establishment of International Emission Trading Mechanism Under The Paris Agreement: Process, Challenge and its Implication for China," Environmental Protection, vol. 13, pp. 58-62, 2021.

[2] National Bureau of Statistics of China, China Statistical Yearbook, China Statistics Press, Beijing, China, 2000-2016.

[3] Ministry of Transport of China, Statistics Bulletin on the Development of the Transportation Industry, 2000-2019.

[4] General Office of the State Council, The Three-Year Action Plan for Promoting the Adjustment of the Transport Structure (2018-2020), 2018, http://www.gov.cn/zhengce/content/201810/09/content_5328817.htm.

[5] National Development and Reform Commission, Guiding Opinions on Accelerating the Construction of Railway Special Lines, 2019, https://www.ndrc.gov.cn/fggz/zcssfz/zcgh/ 201909/t20190918_1195138.html? code=\&state $=123$.

[6] K. Nakamura and Y. Hayashi, "Strategies and instruments for low-carbon urban transport: an international review on trends and effects," Transport Policy, vol. 29, pp. 264-274, 2013.

[7] R. Zhang and Q. Liu, "CO2 emission minimizing for the timedependent VRP in urban Area," Industrial Engineering \& Management, vol. 20, pp. 29-34, 2015.

[8] L. Yang, Y. Cai, X. Zhong, Y. Shi, and Z. Zhang, "A carbon emission evaluation for an integrated logistics system-A case study of the port of Shenzhen," Sustainability, vol. 9, no. 3, p. 462, 2017.

[9] Y. Zhou, W. Fang, M. Li, and W. Liu, "Exploring the impacts of a low-carbon policy instrument: a case of carbon tax on transportation in China," Resources, Conservation and Recycling, vol. 139, pp. 307-314, 2018, https://doi.org/10.1016/ j.resconrec.2018.08.015.

[10] R. Gedik, H. Medal, C. Rainwater, E. A. Pohl, and S. J. Mason, "Vulnerability assessment and re-routing of freight trains under disruptions: a coal supply chain network application," Transportation Research Part E: Logistics and Transportation Review, vol. 71, pp. 45-57, 2014, https://doi.org/10.1016/j.tre. 2014.06.017.

[11] L. Zhao, Y. Zhao, Q. Hu, H. Li, and J. Stoeter, "Evaluation of consolidation center cargo capacity and loctions for China railway express," Transportation Research Part E: Logistics and Transportation Review, vol. 117, pp. 58-81, 2018, https://doi. org/10.1016/j.tre.2017.09.007.

[12] J. Guo, J. Wang, Q. Li, and B. Guo, "Construction of prediction model of neural network railway bulk cargo floating price based on random forest regression algorithm," Neural Computing \& Applications, vol. 31, no. 12, pp. 8139-8145, 2019, https://doi.org/10.1007/s00521-018-3903-5.

[13] D. Marchetti and W. Peter, "Efficiency of the rail sections in Brazilian railway system, using TOPSIS and a genetic algorithm to analyse optimized scenarios," Transportation Research Part E: Logistics and Transportation Review, vol. 135, 2020 https://doi.org/10.1016/j.tre.2020.101858, Article ID 101858.

[14] H. A. Von der Gracht and I.-L. Darkow, "Energy-constrained and low-carbon scenarios for the transportation and logistics industry," International Journal of Logistics Management, vol. 27, pp. 142-166, 2013, https://doi.org/10.1108/IJLM-122013-0150.

[15] S. Turki, S. Didukh, C. Sauvey, and N. Rezg, "Optimization and analysis of a manufacturing-remanufacturing-transportwarehousing system within a closed-loop supply chain," Sustainability, vol. 9, no. 4, p. 561, 2017, https://doi.org/10. 3390/su9040561.

[16] W. Yang, L. Cheng, and L. Li, "Study on the influence of tax restructuring on the profit model of 4th-party logistics platforms," China Business and Market, vol. 30, pp. 100-107, 2016.

[17] W. Zhang, "The Development Trend of China's Logistics Industry in the Era of Internet Plus," Technoeconomics \& Management Research, vol. 4, pp. 104-108, 2018.

[18] P. Wang, A Study of Information Service Paradigm of Government Procurement Cloud Based on Value Chain Collaboration, Tianjin University, Tianjin, China, 2016.

[19] Z.-H. Hu, J.-B. Sheu, and J.-B. Sheu, "Post-disaster debris reverse logistics management under psychological cost minimization," Transportation Research Part B: Methodological, vol. 55, pp. 118-141, 2013, https://doi.org/10.1016/j. trb.2013.05.010.

[20] L. I. Li, F. Cheng, X. Cheng, G. Wang, and T. Pan, "Enterprise remanufacturing logistics network optimization based on modified multi-objective particle swarm optimization algorithm," Computer Integrated Manufacturing Systems, vol. 24, pp. 2122-2132, 2018.

[21] W. Hai-rui, N.-N. Jia, and Z. Lu-ping, "Dry-port-based sustainable logistics network for Inland Provinces in Maritime Silk road," Systems Engineering, vol. 37, pp. 63-73, 2019.

[22] Z. Gu, "An analysis on the demand characteristics of railway bulk goods transport," Railway Transport and Economy, vol. 41, pp. 78-83, 2019.

[23] X. Tao, Q. Wu, and C. Yin, " $\mathrm{CO}_{2}$ reduction potential of shifting containers from road to rail for green transport development," Advances in Climate Change Research, vol. 11, pp. 1-9, 2019.

[24] M. De Gennaro, P. Elena, and G. Martini, "Big data for supporting low-carbon road transport Policiesin Europe application," Big Data Research, vol. 15, pp. 11-25, 2019. 
[25] E. Menezes, A. Gori Maia, A. G. Maia, and C. S. de Carvalho, "Effectiveness of low-carbon development strategies: evaluation of policy scenarios for the urban transport sector in a Brazilian megacity," Technological Forecasting and Social Change, vol. 114, pp. 226-241, 2017, https://doi.org/10.1016/j. techfore.2016.08.016.

[26] T. Kawasaki and T. Matsuda, "Containerization of bulk trades: a case study of US-Asia wood pulp transport," Maritime Economics \& Logistics, vol. 17, no. 2, pp. 179-197, 2015, https://doi.org/10.1057/mel.2014.16.

[27] J. Wu and Y. Wang, "Distribution of the emergency supplies in the COVID-19 pandemic: a cloud computing based approach," Mathematical Problems in Engineering, vol. 2021, pp. 1-18, 2021, https://doi.org/10.1155/2021/5972747, Article ID 5972747.

[28] J. Yang and W. Wan, "Carbon emissions and reduction scenarios of transportation in Jiangsu Province," Highway, vol. 11, pp. 155-159, 2017.

[29] Y. Ding, "Rational Division of Labor and Coordinated Development of Railway and Highway Logistics Transportation," Railway Transport and Economy, vol. 38, pp. 21-25, 2016.

[30] X. Qi, T. He, and B. Mao, "The development and current Status of railway container intermodal transport in China," Journal of Transportation Systems Engineering and Information Technology, vol. 18, pp. 194-200, 2018.

[31] Q. He, W. Luo, and L. Li, "Logistics network planning method research under carbon tax constraint and coexist transport," Journal of Railway Science and Engineering, vol. 11, pp. 131136, 2014. 